\title{
Decoding decisions
}

\author{
How do we select papers for publication in Nature Chemical Biology?
}

n the past four years, Nature Chemical Biology has published original research that covers the breadth of chemical biology. During this time we have been engaged in a dialog with the chemical biology community about the evolution of this expanding discipline and our role in its development. In parallel, we have been committed to refining our editorial processes and expanding the journal to better serve the needs and aspirations of the community. In response to feedback from authors and with an aim to make our editorial decisions more transparent, we would like to explain our editorial processes and highlight some of the common features we seek in Nature Chemical Biology papers.

The manuscript selection process at Nature Chemical Biology differs from that of many chemical biology journals. Like other Naturetitled journals, Nature Chemical Biology has no external editorial board. Instead, editorial decisions are made by a team of professional editors with $\mathrm{PhDs}$ in chemistry and biology (http://www.nature.com/nchembio/ about/about_eds/index.html). Each editor handles a portfolio of chemical biology research areas, and in collaboration with authors, referees and other editors on the team, selects for publication the most significant papers in those subdisciplines. The number of submissions greatly exceeds the number of papers that we can publish, making the journal highly selective. As a result, we feel that it is our responsibility, particularly as a relatively new journal in an emerging field, to be transparent about our processes and to provide clear rationale to authors whose submissions we have declined.

The review process at Nature Chemical Biology includes two steps: editorial assessment and external peer review. We first read and discuss each manuscript in light of our editorial criteria and determine whether the manuscript should be sent for external peer review. For manuscripts that are not initially selected for external review, we contact the authors to decline the paper, and in some cases we encourage resubmission of a revised manuscript. In the latter case, we send the authors a decision letter that contains a web link for submitting a revised paper and encourage them to discuss the study further with the editor. Papers that are selected for external peer review are sent to a panel of scientific referees covering appropriate chemical and biological expertise, who are asked to assess the technical merits and scientific advance of the paper. After review, the editors discuss the manuscript in the context of the referee reports and make a decision to accept or decline the work, or to request a revised manuscript. Most published manuscripts have undergone more than one round of review by the same referees to ensure that all technical concerns have been addressed.

How do we determine which manuscripts are sent for external review? Our first consideration is whether the submitted manuscript falls within the scope of the journal. In principle, chemical biology papers should have a balance of chemical and biological components. However, for most current manuscripts the major advance is focused in one discipline. As a result we consider papers across this spectrum, ranging from fundamental chemical studies that are applied to understanding biological systems to major biological advances that were enabled by chemical approaches. Second, we consider whether the paper reports significant conceptual or methodological advances that are likely to open up new avenues of research in the field. In assessing a manuscript's novelty, we rely on our editorial expertise and familiarity with the field's criteria, but we also closely examine the published literature to provide context for the reported discoveries. Finally, because the journal's audience includes both chemists and biologists, we favor papers that are likely to appeal not only to specialists but also to our broad readership.

Chemical biologists have been consistently successful in applying small molecules, chemical tools and mechanistic thinking to provide fundamental insights into diverse biological systems. This approach remains a principal focus for the field and the journal. In this area, we favor manuscripts that build on their methodological and scientific advances to provide new biological insights under physiologically relevant conditions. For example, we would encourage authors developing new chemical tools to show that their method will be directly useful in addressing important biological questions. In papers describing small-molecule inhibitors, computational and structural approaches or new biochemical pathways, we particularly seek experimental data explaining the mechanism of these systems at the molecular level, and whenever possible, within the native environment of a living cell or organism. More broadly, we are interested in innovative chemical and biological approaches-from synthetic and inorganic chemistry to neuroscience and immunology — that will directly enhance scientists' ability to understand and manipulate biological systems with molecular precision.

In our editorial processes and decisions, we strive to recognize the unique features of each submission and consider the manuscript's merits in the context of the many disciplines that fall within the scope of Nature Chemical Biology. When we communicate our editorial decisions to authors, we provide our assessment of the strengths and limitations of the study and explain our editorial rationale. However, as an author, you should feel free to contact the editor handling the paper if the decision or our rationale is unclear. We are always happy to discuss your manuscript before, during and after submission. We also recognize that because chemical biology is a rapidly evolving field, we must continue to work with the community to ensure that editorial standards are in line with those of the broader disciplines that we serve.

Over the past four years, we have enjoyed getting to know chemical biologists from around the world and hearing about their diverse research. We hope that by keeping the lines of communication open, we can help authors create even more compelling manuscripts and continue to publish top-tier papers covering the entire spectrum of the chemistry-biology interface. 